\title{
Atypical Proximal Limb Pain of Suspected High lumbar Stenotic Origin Arising from SeVere AortoiliaC Disease - Leriche's SYNDROME
}

\author{
Jason S. Lipetz, MD, Jeffry R. Beer, MD, and Jeff S. Silber, MD
}

\begin{abstract}
We present the cases of two middle aged women who were referred to our physiatric spine center with chronic and proximal lower extremity pain complaints of suspected upper lumbar and stenotic origin. Their histories, physical examinations, and imaging studies were not convincing for a primary radicular pain generator. Non-invasive arterial Doppler studies demonstrated a significant
\end{abstract}

brachial to high thigh pressure discrepancy without a focal segmental drop affecting the more distal lower extremity vasculature. Computed tomography (CT) angiograms confirmed advanced aorto-iliac atherosclerotic disease in each case. Spine practitioners are often consulted by the medical community to direct care in patients with suspected lumbar stenosis. These atypical cases of patients with isolated proximal lower extremity pain remind us of the symptomatic overlap between neurogenic and vascular claudication and emphasize the importance of a detailed history, physical examination, and appropriate imaging studies in identifying patients with primary arterial insufficiency.

Keywords: Spinal stenosis, atherosclerosis, arterial insufficiency, claudication.
Symptoms arising from lumbar stenosis and arterial occlusive disease can demonstrate similarities that present a considerable diagnostic challenge to the treating physician. Lumbar stenosis has been classified as either congenital (1) or acquired in origin (2). Compression of the lumbosacral spinal roots can result in radicular symptoms and neurogenic claudication, which typically is worsened with standing and ambulation. Arterial occlusive disease affecting the lower extremities can arise from thrombotic, inflammatory, and spastic disorders of the vasculature. Vascular claudication describes lower extremity pain reproduced through exercise and most commonly arises from atherosclerosis obliterans (3).

We present the atypical cases of two relatively young women referred to a physiatric spine specialist with chronic, proximal lower extremity pain of suspected lumbar and stenotic origin, who ultimately were determined to have symptoms arising from pronounced aorto-iliac occlusive disease.

From Albert Einstein College of Medicine North Shore - Long Island Jewish Health System, New Hyde Park, New York. Address Correspondence: Jason S. Lipetz, MD, North Shore - Long Island Jewish Health System, 801 Merrick Avenue, East Meadow, New York-11554.

E-mail: JEX3@optonline.net

Funding: There was no external funding in preparation of this manuscript.

Conflict of Interst: None

\section{CASE Reports}

\section{Patient A}

A 48-year-old female was referred by her internist with a diagnosis of "spinal stenosis" and the chief complaint of bilateral lower extremity pain. Her symptoms had evolved over the past two years and began without a particular inciting event. A trial of physical therapy had not proven helpful. She described intermittent discomfort affecting the lower lumbar region, but her primary complaint was one of severe aching and burning affecting her anterior thighs, generally not extending beyond the knees. Her lower lumbar discomfort was exacerbated during repetitive forward bending and lifting maneuvers. Her more problematic thigh pain was worsened through prolonged ambulation and could be relieved either by sitting or quiet standing.

Her medications included oral nitrates, a calcium channel blocker, inhalers, an anti-depressant, a recently introduced anti-inflammatory agent, and a daily aspirin. Her past medical history was significant for angina, depression, asthma, and pneumonia. The patient was married homemaker with three children and smoked two packs of cigarettes daily for the past 25 years.

On physical examination, the patient presented as a thin but well nourished female in no apparent distress. Height 5'4, weight 102 lbs., pulse 72, blood pressure 120/70. Muscle girth was symmetrical proximally and distally in the lower extremities. The right posterior tibial pulse was graded trace, and the left was absent. The bilateral popliteal pulses were graded trace. The left femoral pulse was not appreciated and the right was graded trace. There was no pain with pressure applied over the lower lumbar spinous processes. Passive range of motion of her bilateral hips was not pain provoking. Pelvic rocking maneuvers did reproduce her lumbar pain. Root tension signs were negative. She was able to toe and heel walk without difficulty, and no myotomal strength or dermatomal sensory deficits were appreciated. Patellar and Achilles reflexes were graded 2+, as were upper limb reflexes, and no long tract signs were appreciated. Active lumbar flexion did reproduce her low back discomfort. Lumbar extension was performed without resultant axial or limb pain.

She presented with a lumbar magnetic resonance image (MRI) and report as well as plain radiographs performed three weeks prior to her visit. The plain radiographs revealed loss of disc space height at L5-S1, normal alignment in the coronal and sagittal planes, and extensive calcification of the aorta and bilateral iliac vessels. The MRI was read by the interpreting radiologist as demonstrating "thecal sac flattening" at L2-3 and L5-S1. Our review of these images revealed only disc 
desiccation and a small central protrusion with an annular tear at L5-S1. An inspection of the sagittal and axial views did not reveal significant neural foraminal or central canal stenosis.

At the conclusion of her initial evaluation, our differential diagnosis for her debilitating lower extremity complaints included proximal arterial occlusive disease with resultant thigh pain and discogenic low back pain with associated sclerotomal symptom referral. Spinal stenosis and radicular pain were considered much less likely after reviewing her pain distribution and radiographs. Her lumbar pain was felt to be likely arising from a mechanical and discogenic pain generator. To further investigate for a vascular disorder, non-invasive arterial Doppler studies were performed both at rest and after treadmill ambulation with symptom reproduction.

At rest, the right and left ankle/ brachial indexes (ABI) were reduced. The right measured 0.77 (normal $\left.>.90^{4}\right)$ and a more marked reduction was observed on the left where the ABI measured 0.42 (Fig. 1). Segmental pressure measurements revealed a significant reduction in pressure at the high thigh bilaterally, when compared with the brachial pressure measurements, consistent with aorto-iliac or iliofemoral disease. Waveform analysis demonstrated a diffuse reduction in left sided waveform amplitudes with a loss of the dicrotic notch in the right thigh waveforms. A focal area of segmental pressure drop, i.e., decline of 20-30 $\mathrm{mm} \mathrm{Hg}$ (5), was not detected when measuring more distal sites in the thigh, calf, ankle and foot. Exercise treadmill testing was performed for three minutes at an incline of $12 \%$ and a speed of $1.5 \mathrm{mph}$. The patient complained of her typical thigh pain on the left after 90 seconds and on the right after $120 \mathrm{sec}-$ onds. A further decrease of the left ABI to 0.13 and the right $\mathrm{ABI}$ to 0.36 was observed with a return to pre-exercise values after five minutes of rest.

Consultation was arranged with a vascular surgeon. His exam revealed a right carotid bruit. The patient was scheduled for a CT angiogram, as she was claustrophobic and refused an MRI angiogram, and carotid Doppler. The CT angiogram revealed diffuse atherosclerotic disease involving the distal abdominal aorta with extensive calcifications and mural thrombus formation. Bilateral common iliac artery stenosis was observed at the bifurca-

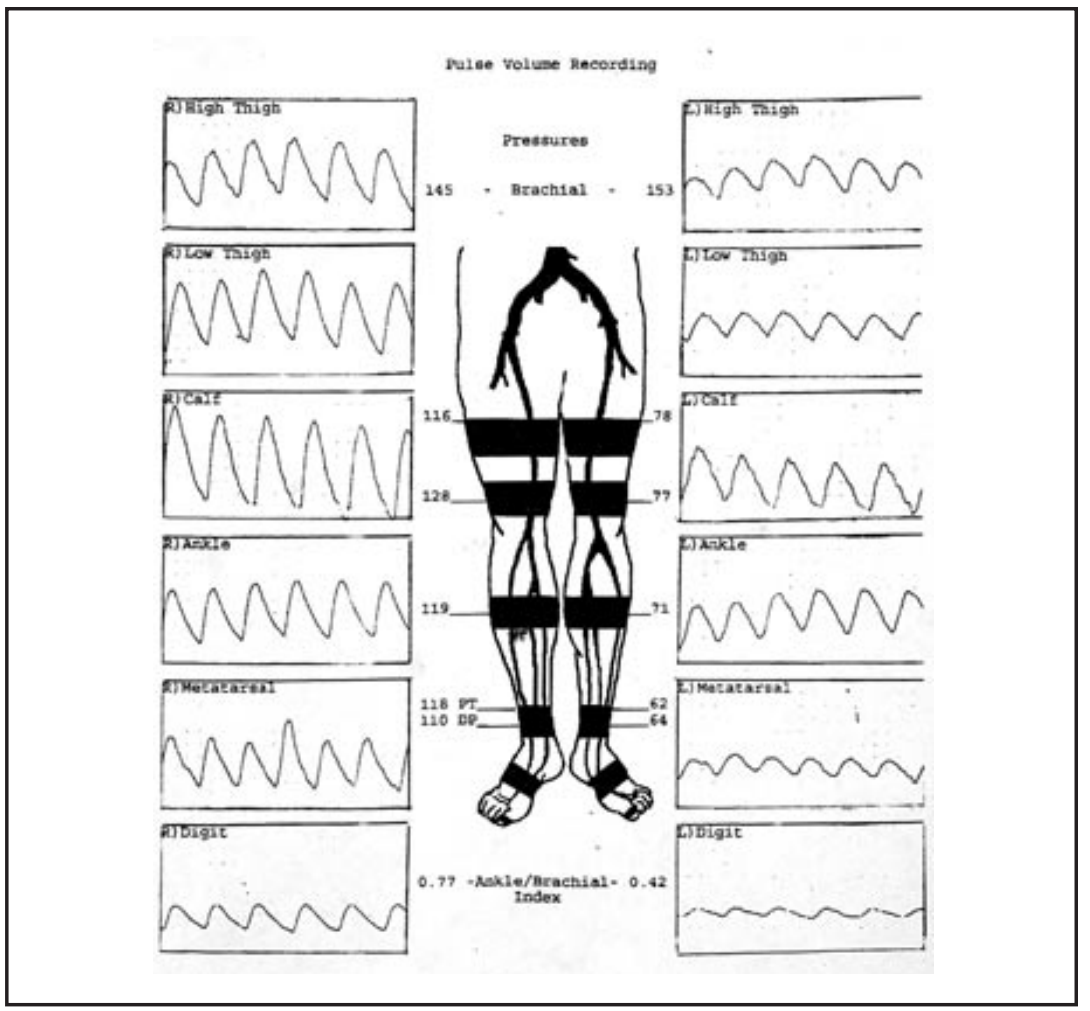

Fig 1. Segmental pressure readings obtained from patient $A$ 's lower extremities at rest. Note the reduction in the bilateral high thigh pressures when compared to the brachial values. In normals, an increase of $30-40 \mathrm{mmHg}$ is observed from the brachial to the proximal femoral artery (4). A more marked reduction in the left lower extremity waveform amplitudes is observed.

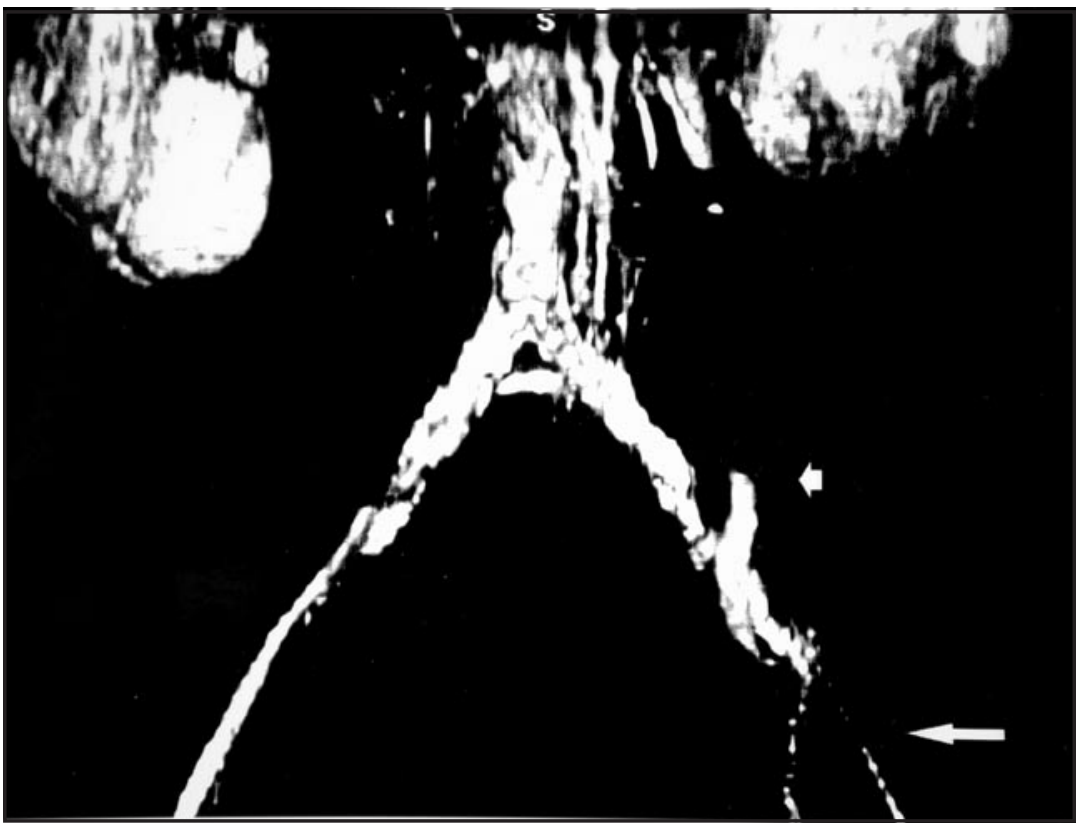

Fig 2. CT Angiogram demonstrating severe stenosis of the left external iliac artery (long arrow) and a suspected arteriovenous malformation (short arrow) within the left pelvis 
tion. Diffuse atherosclerosis of the bilateral common and external iliac arteries was observed with a discrete short area of stenosis affecting the proximal right external iliac and more severe and diffuse disease on the left where a short segmental occlusion of the external iliac artery was observed (Fig. 2). The left common femoral artery demonstrated a calcific mural thrombus, and moderate stenosis was noted to affect the mid portion of the right superficial femoral artery.

Carotid Doppler revealed complete occlusion of the left internal carotid and findings consistent with $99 \%$ stenosis of the right internal carotid from a likely more recently formed soft plaque. The patient underwent a successful right carotid endarterectomy. She remained neurologically intact. Her lower extremity symptoms were initially treated with an oral anti-platelet agent. If after two months of treatment her symptoms were found to persist in a debilitating fashion, her candidacy for bilateral aorto-femoral bypass was to be further reviewed.

\section{Patient B}

A 57-year-old female was referred by her internist with a working diagnosis of "lumbar stenosis" and the chief complaint of proximal bilateral lower extremity pain. Her symptoms had evolved over the past three years and began without a particular inciting event. Multiple trials of physical therapy had not offered lasting relief. Her past history was significant for polio and a thoracolumbar fusion performed during her early teenage years for a progressive scoliosis. She denied significant lumbar pain and reported severe bilateral and symmetric anterior and posterior thigh pain, with symptoms not extending beyond the knees. Her thigh complaints were worsened with prolonged ambulation and could be relieved either by sitting or quiet standing.

Her only medication was an anti-resorptive agent prescribed for osteoporosis. Her past medical history was otherwise significant for polio, which predominantly affected the thoracolumbar truncal musculature. Her family history was significant for paternal coronary artery disease. The patient was married with two children, was a housewife, and discontinued smoking two years prior, following a sixty pack-year history.

The patient presented as a wellnourished female in no apparent distress.
Height was 5'6, weight $145 \mathrm{lbs}$., pulse 86, blood pressure 130/80. Muscle girth was symmetrical proximally and distally in the lower extremities. Bilaterally, posterior tibial and dorsalis pedis pulses were absent. Capillary refill at the great toe did not appear delayed bilaterally. The bilateral popliteal and femoral pulses were graded trace. There was no pain with pressure applied over the lower lumbar spinous processes. Passive range of motion of the bilateral hips was not pain provoking. Pelvic rocking, sustained hip flexion, and root tension maneuvers were also not provocative. She was able to toe and heel walk without difficulty, and no myotomal strength or dermatomal sensory deficit was appreciated. Patella and Achilles reflexes were graded $2+$, as were upper limb reflexes, and no long tract signs were appreciated. Active lumbar flexion and extension were not pain provoking.

She presented with a lumbar MRI that was performed eight months prior to her visit. Our review of these images revealed disc desiccation and advanced loss of disc space height and type-I end plate reactive change at L3-4 and L4-5, moderate neural foraminal stenosis on the right at L4 and L3, a foraminal disc protrusion to the right at L4-5, posterior element arthrosis at L2-3, and mild central canal compromise at L3-4.

Also available for review was the report from a lower extremity arterial Doppler exam performed two years earlier that revealed "no vascular occlusion or area of high grade stenosis within the arterial system of the right or left lower extremity from the level of the external iliac artery to the proximal trifurcation vessels of the calf." An electrodiagnostic study performed one-year prior revealed normal distal sensorimotor amplitudes and no evidence of denervation in the paraspinal or lower extremity musculature.

At the conclusion of her initial evaluation, our differential diagnosis included proximal arterial occlusive disease with resultant thigh pain, symptomatic lumbar foraminal stenosis with associated radicular pain, and a variant of post-po-

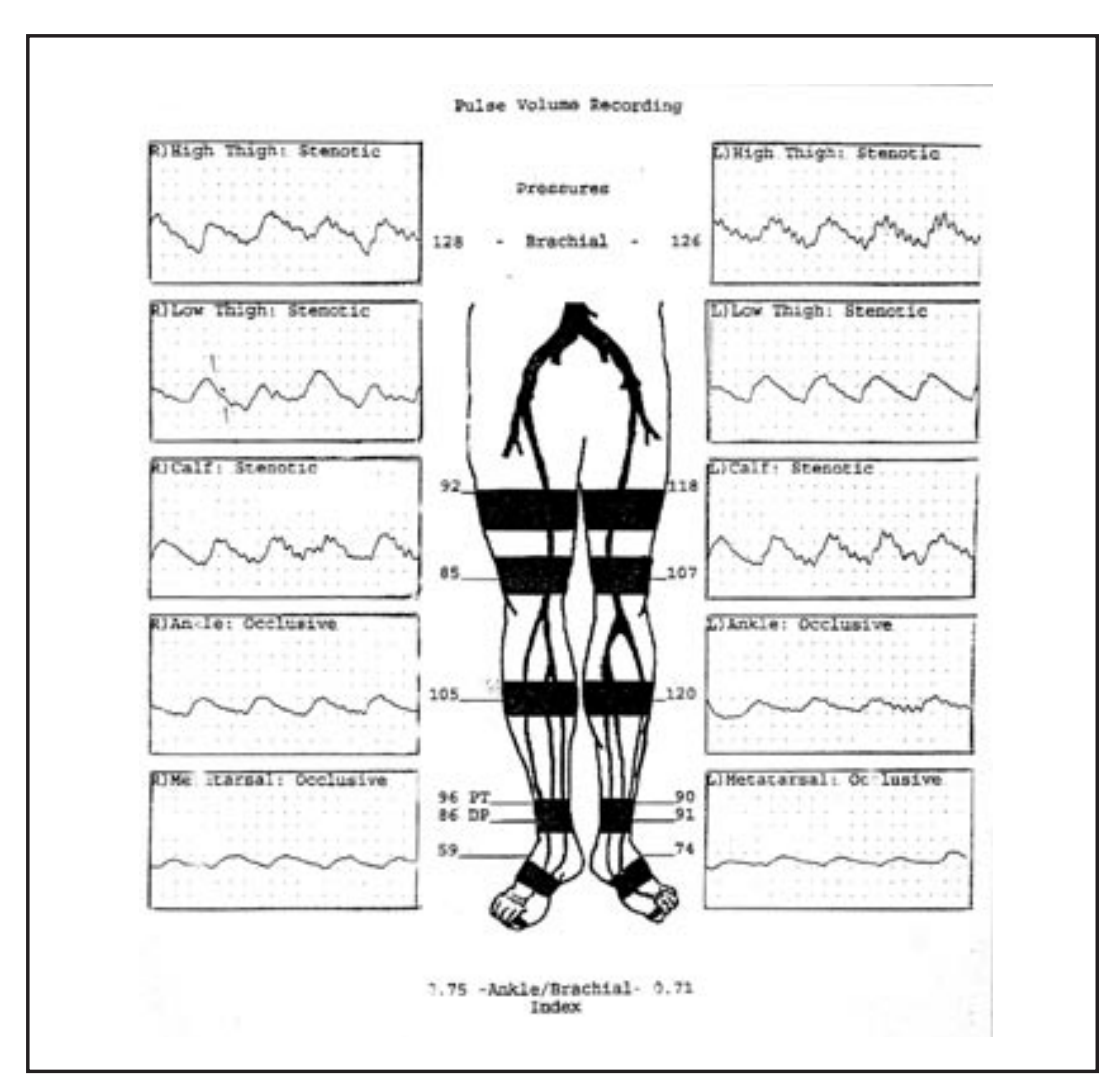

Fig 3. Segmental pressure readings obtained from patient $B$ 's lower extremities at rest. Again, note the reduction in the bilateral high thigh pressures when compared to the brachial values. A more pronounced reduction in the lower extremity waveform amplitudes is appreciated bilaterally 
lio syndrome. There were several problems with the assignment of lumbar stenosis as a primary diagnosis. Her foraminal compromise was right sided only, and while involvement of the L3 and L4 nerve roots could result in ipsilateral right sided anterior thigh pain, her pain complaints were bilateral and tended to affect the proximal thighs in a more circumferential fashion. There was no significant central canal compromise or stenosis of the contralateral foramen to account for her left lower extremity pain. Additionally, her pain could be relieved through rest and quiet standing without the need to sit or assume a forward flexed posture. She also reported a significant smoking history, and to further investigate for a vascular disorder, non-invasive arterial Doppler studies were ordered. It was similarly requested that these be performed both at rest and with symptom reproduction following treadmill ambulation.

Secondary to insurance restrictions, the patient was only able to have her studies performed at an outside hospital, and studies were only performed at rest. The right $\mathrm{ABI}$ was reduced and measured 0.75 and the left ABI measured 0.71 (Fig. 3). Segmental pressure measurements revealed a significant reduction in pressure at the high thigh bilaterally, when compared with the brachial pressure measurements, consistent with aorto-iliac or iliofemoral disease. Waveform analysis demonstrated a diffuse reduction in waveform amplitudes bilaterally. A focal area of segmental pressure drop was not detected when measuring more distal sites in the thigh, calf, ankle and foot.

Consultation was arranged with a vascular surgeon. A CT angiogram was obtained and revealed severe narrowing of the aortic lumen at a level $6 \mathrm{~cm}$ below the renal arteries, with pronounced narrowing extending through the iliac bifurcation and the bilateral internal iliac arteries. Significant stenosis and atherosclerotic change of the more distal lower extremity vasculature was not appreciated. The patient underwent a carotid Doppler exam, which demonstrated patent vessels. A cardiac stress test did not reveal significant coronary disease.

\section{DisCUSSION}

Symptoms of spinal stenosis and neurogenic claudication typically occur after walking a short distance or standing for a prolonged period and are described as a sensation of pain, weakness, heaviness or tiredness of the lower extremities which forces the patient to stop walking or change position. It has been suggested that the point at which the patient stops ambulating is typically approximately twice the distance at which the lower extremity complaints begin. Symptoms of spinal stenosis can vary during the day and from day to day. Patients find relief through reducing walking speed, assuming a forward flexed posture, or sitting (1, $6,7)$. Patients presenting with symptoms of neurogenic claudication will also often describe an antecedent and long-standing history of low back pain (7-9).

Vascular claudication has been described as a sensation of muscle cramping affecting the calf or thigh. Symptoms typically arise after the patient has walked a defined distance and resolve through resting in the standing position after a predictable period of time $(10,11)$. Vascular claudication complaints are experienced in a functional muscle unit, are incited through exercise, and are relieved through a discontinuation of the exercise, without the need for postural change (12). While the patient's history will often guide the treating physician in identifying a vascular as opposed to a spinal stenosis presentation, the primary diagnosis may not always be clear. Particular confusion can arise in those patients with coexistent vascular insufficiency and degenerative spinal disease (13).

The most common cause of arterial occlusive disease is atherosclerosis obliterans. Atherosclerotic plaques have been shown to affect the arterial tree in a segmental fashion and most often at points of bifurcation, areas where arteries follow a more tortuous course, or zones of more rapid vessel tapering (14). While atherosclerosis tends to occur with equal frequency in both the femoral and tibial arteries in diabetics, in non-diabetic patients the most common sites of involvement are the aorta, iliac, and femoral arteries (15). The syndrome of aortoiliac occlusive disease, also known as Leriche's syndrome, was first described in 1923 in young male smokers $(16,17)$. To this day, the primary risk factor for arterial occlusive disease remains smoking. The increased risk of claudication has been calculated to be 15 times higher in male smokers compared with non-smokers and seven times higher in female smokers than in their non-smoking counterparts
$(18,19)$. The increased risk is directly related to the number of cigarettes smoked, and the risk increase is thought to persist for up to five years following smoking cessation $(20,21)$. Hypertension, diabetes, obesity, older age, and hyperlipidemia also represent significant risk factors for claudication, and the presence of multiple risk factors further increases the risk of arterial occlusive disease (19-22).

The pain of vascular claudication arises from the muscle groups distal to the site of arterial obstruction. Symptoms are believed to arise during exercise from inadequate blood flow to the muscle tissue with resultant ischemia and an increase in metabolic waste products $(23,24)$. At rest, blood flow may be adequate to muscles downstream from the site of occlusion secondary to a reduced metabolic demand and contributions from enlarged collateral vessels. In both of our patients, the initial diagnostic test performed was a non-invasive segmental Doppler study. In addition to the reduced ABI observed in each case at rest, the segmental pressure readings obtained from the proximal and distal lower extremity were of particular interest. In both cases, diffusely blunted waveforms were appreciated with a loss of the typical dicrotic notch, but a localized significant drop in pressure measurement was not appreciated between any adjacent limb segments. A significant drop was only realized when comparing the proximal thigh reading to that obtained from the brachial artery. Normal values during Doppler studies reveal an increase of $30-40 \mathrm{mmHg}$ from the brachial to the proximal femoral artery reading (4). In our second patient, previously performed arterial Doppler did not reveal occlusion when investigating the iliac to more distal calf vasculature. Without segmental pressure studies comparing the proximal thigh to brachial pressure values, a diagnosis of more proximal aorto-iliac disease was missed.

While resting studies were quite informative in each of our patients with more advanced occlusive disease, exercise studies can be quite helpful in identifying patients with more borderline arterial insufficiency and less pronounced resting pressure abnormalities. During exercise, and in the setting of occlusive disease, distal arteries dilate in an attempt to augment regional blood flow with a resultant decrease in pressure readings (25). On oc- 
casion, a patient with a history suggestive of vascular claudication will demonstrate normal distal pulses and ABI's at rest, and only through exercise with repeated pressure measurements will arterial obstruction be revealed.

The differentiation between symptoms arising from vascular and neurogenic claudication can be particularly challenging in patients presenting with coexistent arterial occlusive and degenerative spine disease. Our first patient presented with a significant mechanical lumbar pain component which might be ascribed to her degenerative disc disease and annular tear at L5-S1. Our second patient also presented with a significant surgical spine history and radiographic findings demonstrative of multiple levels of degenerative and foraminal stenosis. A diagnosis of arterial insufficiency might also have been considered less likely as each patient's pain involved the proximal lower extremities rather than the calves. In each case, the provocative history and examination were suggestive of a vascular presentation, and the observed spinal pathology was ultimately determined not to represent the primary pain generator.

A review of the literature has suggested a further twist in the interplay between vascular and spinal disease. A third variant of claudication, neurogenic claudication arising secondary to vascular disease, has been described (26-28). In these patients, typical symptoms of neurogenic claudication are believed to arise not from spinal stenosis but rather from aortic occlusive disease and intermittent ischemia of the distal spinal cord and cauda equina.

The first patient proceeded with a prompt carotid endarterectomy prior to addressing her lower extremity disease. This case serves as a particular reminder of the importance of performing a complete vascular and cardiac evaluation in patients with newly diagnosed peripheral vascular disease. The medical risk factors associated with claudication are also related to other complications of atherosclerosis. The risk of death from all causes has been estimated to be four to seven times higher in patients with vascular claudication, and patients with claudication have demonstrated 10-year mortality rates from cardiovascular disease up to 15 times higher than age matched controls $(25,29)$.

Author Affiliation:
Jason S. Lipetz, MD
Assistant Professor
Department of Rehabilitation
Medicine
Albert Einstein College of Medicine
Director
Center for Spine Rehabilitation
North Shore - Long Island
Jewish Health System
801 Merrick Avenue
East Meadow, New York-11554
E-mail: JEX3@optonline.net
Jeffry R. Beer, MD
PGY-4 Resident
Division of Rehabilitation Medicine
North Shore - Long Island
Jewish Health System
270-05 76th Avenue
New Hyde Park, New York-11042
Jeff S. Silber, MD
Spine Surgery
Department of Orthopedic Surgery
North Shore - Long Island Jewish
Health System
270-05 76th Avenue
New Hyde Park, New York-11042
New

\section{REFERENCES}

1. Verbiest H. A radicular syndrome from developmental narrowing of the lumbar intervertebral canal. J Bone Joint Surg 1954; 36:230-237.

2. Towne EB, Reichert FL. Compression of the lumbosacral roots of the spinal cord by thickened ligamenta flava. Ann Surg 1931; 94:327-336.

3. Abramson D. Arterial disorders of the extremities: Clinical syndromes, assessment and measurement. Arch Phys Med Rehabil 1968; 49:315-325.

4. Strandness DE Jr. Noninvasive vascular laboratory and vascular imaging. In Young JR, Olin JW, Bartholomew JR et al (eds). Peripheral Vascular Diseases. Mosby Year Book, St Louis, 1991:36-69.

5. Branes RW. Noninvasive diagnostic assessment of peripheral vascular disease. Circulation 1991; 83:20-27.

6. Porter RW. Spinal stenosis and neurogenic claudication. Spine 1996; 21:20462052.

7. Turner JA, Ersek M, Herron L et al. Surgery for lumbar spinal stenosis. Attempted meta analysis of the literature. Spine 1992; 17:1-8.

8. Katz JN, Dalgas M, Stucki G et al. Degenerative lumbar spinal stenosis. Diagnostic value of the history and physical examina- tion. Arthritis Rheum 1995; 38:1236-1241.

9. Katz JN, Dalgas M, Stucki G et al. Diagnosis of lumbar spinal stenosis. Rheum Dis Clin North Am 1994; 20:471-483.

10. Rose GA. The diagnosis of ischemic heart pain and intermittent claudication in field surveys. Bull World Health Organ 1962; 27:645-648.

11. Warren R. Two kinds of intermittent claudication (Editorial). Arch Surg 1976; 111: 739.

12. Kempczinski RF. The differential diagnosis of intermittent claudication. Pract Cardiol 1981; 7:53-57.

13. Stanton PE Jr, Rosenthal D, Clark M et al. Differentiation of vascular and neurogenic claudication. The Amer Surg 1987; 53:71-76.

14. Strandness DE Jr, Langlois YE, Roederer GO. Preoperative evaluation of vascular Disease. In Haimovici H (ed). Vascular Surgery: Principles and Techniques, $2^{\text {nd }}$ ed. Aplleton-Century-Crofts, Norwalk, 1984:43-64.

15. Juergens JL, Spittell JA Jr, Fairbairn JF II. Peripheral Vascular Disease, $5^{\text {th }}$ ed.. WB Saunders, Philadelphia, 1980.

16. Leriche R. Des obliterations arterielles hautes (obliteration de la terminaison de l'aorte) comme causes des membres inferieurs. Bull Mem Soc Chir Paris 1923; 49: 104-106.

17. Leriche R. De la resection du carrefour aortico-iliaque avec double sympathectomie lombaire pour thrombose arteritique de l'aorte le syndrome de l'obliteration termino-aortique par arterite. Presse Med 1940; 48:601-604.

18. Andrews KL, Rooke TW, Helm PA. Rehabilitation in Vascular Diseases. In Braddom RL (ed). Physical Medicine and Rehabilitation. WB Saunders, Philadelphia, 1996: 1180-1197.

19. Ernst CB, Stanley JC. Therapy in Vascular Surgery, $2^{\text {nd }}$ ed. BC Decker, Philadelphia, 1991.

20. Bowlin SJ, Medalie JH, Flocke SA et al. Epidemiology of intermittent claudication in middle-aged men. Am J Epidemiol 1994; 140:418-430.

21. Kannel WB, McGee DL. Update on some epidemiologic features of intermittent claudication: the Framingham study. J Am Geriatr Soc 1985; 33:13-18.

22. Ekroth R, Dahllof AG, Gundevall B. Physical training of patients with intermittent claudication: Indications, methods, and results. Surgery 1978; 84:640.

23. Hiatt WR, Stoll S, Nies AS. Effect of betaadrenergic blockers on the peripheral circulation in patients with peripheral vascular disease. Circulation 1985; 72:1226-1231.

24. Gardner AW. Dissipation of claudication pain after walking: implications for endurance training. Med Sci Sports Exerc 1993; 25:904-910.

25. Santilli JD, Rodnick JE, Santilli SM. Claudication: Diagnosis and treatment. Am Fam Phys 1996; 53:1245-1253.

26. Murphy MA, Denton MJ, Scott DF. Neuro- 
genic claudication secondary to vascular disease. Aust N Z J Surg 1992; 62:154-157.

27. Dejerine J. La claudication intermittente de la moelle epinier. Presse Med 1911; 19:
981-984.

28. Ratinou G, Jimeny-Palon E. Intermittent spinal ischaemia. Neurology 1961; 7:546-549.
29. Criqui MH, Langer RD, Fronek A et al. Mortality over a period of ten years in patients with peripheral artery disease. $N$ Engl J Med 1992; 326:381-386. 